\title{
Mental and physical burdens in a hot environment: Assessments of work clothes with an air cooling function and cold compresses attached to the neck
}

\author{
Shimpei Yamada (sin-y@health.uoeh-u.ac.jp) ${ }^{1}$, Yuto Fukuyama (z134417@info.uoeh-u.ac.jp) ${ }^{1}$ \\ ${ }^{1}$ School of Health Sciences, University of Occupational Environmental Health, Kitakyushu 807-8555, Japan,
}

\begin{abstract}
The effect of Air-Conditioned Jacket and cold pack was investigated in hot environment (35 degree Celsius) by time domain HRV parameters and core temperature, etc. The cooling wear and cold pack did not affect on work performances and core temperature. However, HR, skin temperature and perspiration were decreased by them. Poincare plot SD1, NN50 and pNN50 were significantly larger in the cooling condition than the no-cooling condition.
\end{abstract}

Keywords: Heart rate, Core temperature, Poincare plot

\section{Introduction}

With the influence of global warming, it has been predicted that the incidence of people experiencing heatstroke will increase. Physiological and subjective indicators were measured to examine the effects of work clothes with an air cooling function and cold compresses attached to the neck during work in a hot environment. Relationships between physiological indicators in a hot environment were also investigated from the correlation coefficients between them.

\section{Method}

The study subjects were 10 male university students $(21.3 \pm 1.3$ years old $)$ to whom the experiment was explained in writing in advance, following which their informed consent was obtained. This study was approved by the ethics committee of the University of Occupational and Environmental Health.

The subjects were directed to an artificial climate chamber and then fitted with electrocardiogram electrodes as well as a probe to measure rectal temperature. Sensors to measure skin temperature were attached to the forehead, chest and back. The subjects performed exercise on a bicycle ergometer set to a load of $80 \mathrm{~W}$ (TASK) in a controlled environment at a temperature of $35^{\circ} \mathrm{C}$ and $50 \%$ relative humidity. In parallel with the ergometer exercise, they were given mental arithmetic tasks. Subjects were instructed to remain in a seated position with their eyes open (REST) for 10 min before exercise and 30 min after exercise at a temperature of $27^{\circ} \mathrm{C}$.

Before and after rest and after exercise, subjective assessments were made using a 7-step scale for heat sensation (1: cold $\sim 7$ : hot), feeling of fatigue (1: not tired at all $\sim 7$ : very tired), and body temperature (1: low body temperature $\sim 7$ : high body temperature). The work load after exercise was assessed using the National Aeronautics and Space Administration-Task Load Index (NASA-TLX).

The exercises were performed under three conditions on separate days, namely use of both work clothes with an air cooling function and a cold compress worn on the neck, use of only a cold compress on the neck, and no measures against the heat. The order of the experiments was counter-balanced in consideration of an order effect. The cold compress was attached and the air cooling clothes put on just before the start of exercise.

The RR interval was calculated from the electrocardiogram, and the coefficient of variation of $R-R$ interval $\left(\mathrm{CV}_{\mathrm{R}-\mathrm{R}}\right)$, number of intervals of successive $\mathrm{R}-\mathrm{R}$ intervals greater than $50 \mathrm{~ms}$ (NN50), pNN50 (NN50 / (total number of R-R intervals)), and heart rate (HR) were obtained. A Poincare plot of the R-R intervals was made, and the feature quantity (SD1, SD2) was calculated (Kamen et al., 1996). The measured data were divided into 10-min blocks (REST1, TASK 1 3, REST 2 4) for which mean values were calculated.

The NASA-TLX subscale scores (mental demand, physical demand, temporal demand, own performance, effort, frustration level) and weighted workload were calculated. Amount of change in body weight before and 


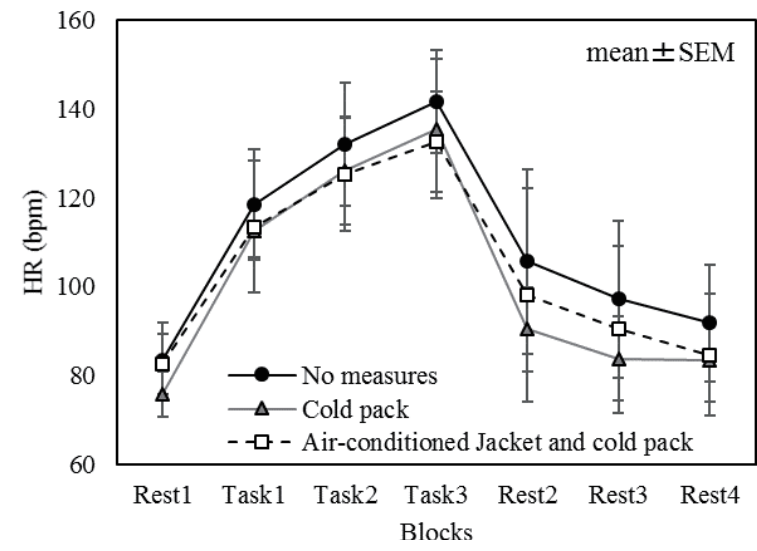

Figure 1. Changes in heart rate (HR)

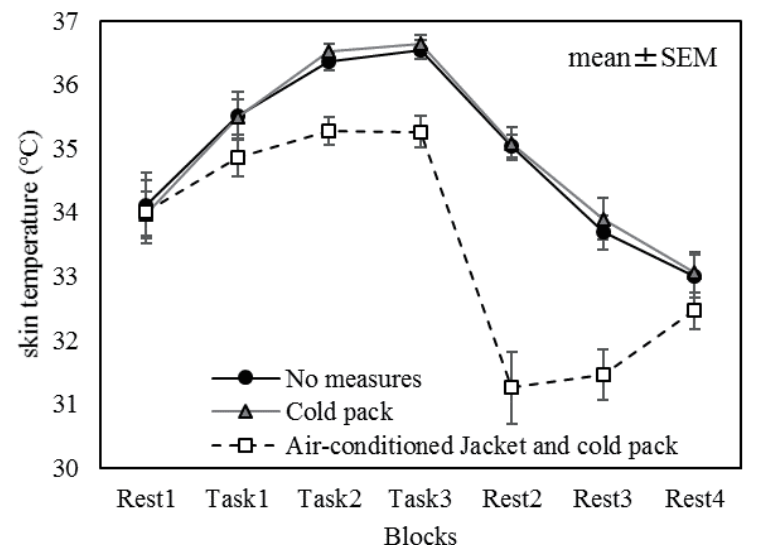

Figure 2. Changes in chest skin temperature

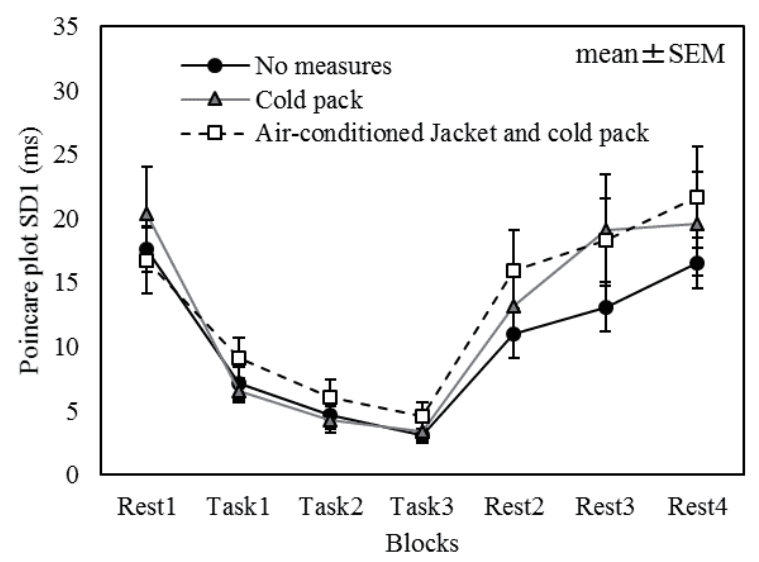

Figure 3. Changes in Poincare plot SD1

after the experiment and amount of perspiration were estimated from the amount of water consumed.

Two-way ANOVAs for conditions and blocks were carried out for comparisons of $\mathrm{CV}_{\mathrm{R}-\mathrm{R}}$, NN50, pNN50, HR, rectal temperature, skin temperature, amount of perspiration and subjective assessments. Correlation coefficients between physiological indicators were calculated. The significance level was set at $\mathrm{p}<0.05$.

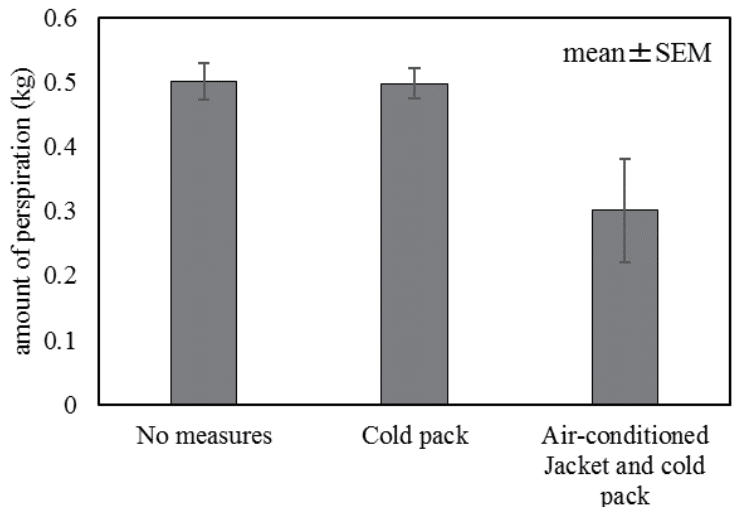

Figure 4. Changes in amount of perspiration

\section{Results}

Significant main effects of the cooling measures were observed in HR, skin temperature, and SD1 (Figs. 1-3). HR and skin temperature were low with use of the air-conditioned jacket and cold pack, while SD1 was high with no cooling measures.

RR interval, $\mathrm{CV}_{\mathrm{R}-\mathrm{R}}$, NN50, pNN50, and Poincare plot feature quantity, which are indicators of parasympathetic nerve activity, showed a significant positive correlation with use of the cooling measures.

\section{Conclusion}

This study showed that working in hot environments may inhibit elevations in heart rate as well as increases in chest and back skin temperature. Perspiration was shown to be decreased with combined use of a cold compress on the neck and air cooling clothes. The results also suggested that a sense of fatigue and body temperature elevation can be prevented by these cooling methods. The Poincare plot quantities, NN50, and pNN50, were significantly higher under the cooling condition than in the no anti-heat measures condition. The results also suggested that these measures do not inhibit a rise in rectal temperature.

\section{References}

Kamen PW, Krum H, Tonkin AM., Poincaré plot of heart rate variability allows quantitative display of parasympathetic nervous activity in humans., Clinical Scienc, 91(2), 201-208, 1996. 